\title{
Multi-Zone Thermodynamic Modelling of Spark-Ignition Engine Combustion - an Overview
}

\author{
${ }^{*}$ S. Verhelst ${ }^{a}$ and C.G.W. Sheppard ${ }^{b}$ \\ ${ }^{a}$ Department of Flow, Heat and Combustion Mechanics, Ghent University \\ Sint-Pietersnieuwstraat 41, B-9000 Gent, Belgium \\ ${ }^{\mathrm{b}}$ School of Mechanical Engineering, The University of Leeds, \\ Leeds LS2 9JT
}

*corresponding author: T +32 9264 3306; F +32 9264 3590; E-mail Sebastian.Verhelst@UGent.be

\begin{abstract}
Multi-zone thermodynamic engine model' is a generic term adopted here for the type of model also referred to as quasi-dimensional, two-zone, three-zone, etc.; based on the laws of mass and energy conservation and using a mass burning rate sub-model (as opposed to a prescribed mass burning rate) to predict the in-cylinder pressure and temperature throughout the power cycle. Such models have been used for about three decades and provide valuable tools for rapid evaluation of the influence of key engine parameters. Numerous papers have been published on the development of models of varying complexity and their application. The current work is not intended as a comprehensive review of all these works, but presents an overview of multi-zone thermodynamic models for spark ignition engines, their pros and cons, the model equations and sub-models used to account for various processes such as turbulent wrinkling, flame development, flame geometry, heat transfer etc. It is suggested that some past terminology adopted to distinguish combustion models (e.g. 'entrainment' versus 'flamelet') is artificial and confusing; it can also be difficult to compare the different models used. Naturally, different models use varying underlying assumptions; however, the influence of several physical processes have frequently been incorporated into one term, not always well documented or clearly described.

The authors propose a unified framework that can be used to compare different sub-models on the same basis, with particular focus on turbulent combustion models.
\end{abstract}

Keywords: internal combustion engine, spark ignition, thermodynamic, modelling, simulation

\section{Nomenclature}

Symbols
A $\quad$ area $\left(\mathrm{m}^{2}\right)$
$A_{f} \quad$ flame surface area $\left(m^{2}\right)$
$D_{T} \quad$ thermal diffusivity $\left(\mathrm{m}^{2} / \mathrm{s}\right)$
C calibration constant (-)
$\mathrm{C}_{\mathrm{v}} \quad$ specific heat at constant volume $(\mathrm{J} / \mathrm{kgK})$
$c_{p} \quad$ specific heat at constant pressure $(\mathrm{J} / \mathrm{kgK})$ 


\begin{tabular}{|c|c|}
\hline $\mathrm{h}$ & specific enthalpy $(\mathrm{J} / \mathrm{kg})$ \\
\hline $\mathrm{I}_{0}$ & stretch factor (-) \\
\hline I & length scale (m) \\
\hline $\mathrm{m}$ & mass $(\mathrm{kg})$ \\
\hline$p$ & pressure $(\mathrm{Pa})$ \\
\hline Q & heat loss $(\mathrm{J})$ \\
\hline$r_{f}$ & flame radius \\
\hline $\mathrm{R}$ & specific gas constant (J/kgK) \\
\hline $\mathrm{t}$ & time \\
\hline $\mathrm{T}$ & gas temperature $(\mathrm{K})$ \\
\hline u & specific internal energy $(\mathrm{J} / \mathrm{kg})$ \\
\hline$u^{\prime}$ & rms turbulent velocity (m/s) \\
\hline $\mathrm{u}_{1}$ & laminar burning velocity $(\mathrm{m} / \mathrm{s})$ \\
\hline $\mathrm{u}_{\mathrm{n}}$ & stretched laminar burning velocity $(\mathrm{m} / \mathrm{s})$ \\
\hline $\mathrm{u}_{\mathrm{t}}$ & turbulent burning velocity $(\mathrm{m} / \mathrm{s})$ \\
\hline U & internal energy $(\mathrm{J})$ \\
\hline V & volume $\left(\mathrm{m}^{3}\right)$ \\
\hline W & work (J) \\
\hline
\end{tabular}

\section{Greek Symbols}
$\delta_{\mathrm{t}} \quad$ turbulent flame thickness $(\mathrm{m})$
$\theta \quad$ crank angle $\left({ }^{\circ} \mathrm{Ca}\right)$
$\rho \quad$ gas density $\left(\mathrm{kg} / \mathrm{m}^{3}\right)$
$\tau \quad$ time constant (s)

\section{Subscripts}

$\begin{array}{ll}\text { b } & \text { burned } \\ \text { e } & \text { entrained } \\ \text { I } & \text { integral } \\ \text { K } & \text { Kolmogorov } \\ \text { I } & \text { 'leakage' (blow-by) } \\ \text { r } & \text { 'reacted' }\end{array}$




$\begin{array}{ll}\mathrm{T} & \text { Taylor } \\ \mathrm{u} & \text { unburned }\end{array}$

Abbreviations

$\begin{array}{ll}{ }^{\circ} \mathrm{Ca} & \text { degrees crank angle } \\ \text { EGR } & \text { exhaust gas recirculation } \\ \text { IVC } & \text { inlet valve closing } \\ \text { TDC } & \text { top dead center }\end{array}$

\section{Introduction}

Shown in Fig. 1 is a photograph of a Rapid Prototype 3-D reconstruction of (part of) a turbulent flame at low rms turbulent velocity in the Leeds Mk2 fan-stirred constant volume bomb (with 3-D coordinates drawn from multiple mie-scatter laser sheet images generated using a "swinging sheet" technique [1]). Correct modelling of the propagation of a flame of such geometric complexity, with its numerous irregular protrusions, troughs and folds, is problematic but necessary in order properly to understand the combustion process and so assist design, development and optimization of engines.

Several model frameworks are used for the simulation of the ('closed' part of the) spark ignition engine cycle, these can be classified as 'zero-', 'multi-zone' and 'multi-dimensional' models. The first two types are classified as thermodynamic models, where the equations constituting the basic structure of the model are based on conservation of mass and energy and are only dependent on time (resulting in ordinary differential equations). Multi-dimensional models are also termed fluid mechanic or fluid dynamic models, where the governing equations are the Navier-Stokes equations in addition to conservation of mass and energy (the equations are also dependent on the spatial coordinates, and so take the form of partial differential equations).

Multi-zone models are distinguished from zero-dimensional models by the inclusion of certain geometrical parameters in the basic thermodynamic approach. This usually involves the radius of a thin interface (the flame) separating burned from unburned gases, resulting in a 'two-zone' formulation. Zero-dimensional models; also termed single-zone models; use a predefined mass burning rate, of which the Vibe law (in English literature normally referred to as the Wiebe law) is the best known example. This mass burning rate has to be empirically defined for every engine operating point, on the basis of prior engine experiments or experience and is not expressed in terms of physical quantities (e.g. fuel properties, engine geometry etc.) rendering extrapolation to other operating conditions problematic. However, where such data are available or require little extrapolation, this can be the best approach, as it avoids modelling of the in-cylinder processes and as it, in effect, works back from a known result and hence should provide unrivalled accuracy in predictions.

Where such data are unavailable, for instance for exploring wide ranges of new engine concepts (pressure charging, intercooling, variable valve timing - e.g. to run Miller/Atkinson type cycles, EGR, multiple spark plugs etc.) which are too complex for the design\&development engineer to have an 
intuitive grasp on how they will affect the combustion rate, the mass burning rate must be modelled. This is done in both multi-zone or multi-dimensional models. The choice of multi-zone or multidimensional model is largely determined by the application. If the objective is to evaluate a large range of conditions, perform parametric studies and/or predict optimum engine settings, a reasonable accuracy and fast computation on a PC system is desirable. These conditions are satisfied by multizone models. Recent examples are the investigations of causes for cycle-to-cycle variations in engines [2] and causes for the increased combustion variability leading to lean limits [3. Multidimensional models are inappropriate for such studies as they are computationally too demanding. Their best use is for more detailed studies for limited conditions or particular features (e.g. flow through valves, fuel injection, bulk in-cylinder flow and turbulence development), or to support theory and model development.

As discussed later, a mass burning rate model should take account of the turbulent flame brush thickness, representing the distance between the leading ('protusions') and trailing edge ('troughs') of the turbulent flame (which can be defined in several ways, see ref. [4]). Models that provide for this are also sometimes termed three-zone models, with the 'entrained but not (yet) burned' zone conceptually a third zone in addition to the fully burned and fully unburned zones.

The objective of this paper is to present a critical overview of modelling spark ignition engine combustion using the multi-zone thermodynamic approach. Such models were the subject of an earlier excellent review paper by Blumberg et al. published in 1979 [5]. However, given new insights and the better understanding of engine combustion and combustion in general, it is considered timely to offer a contemporary review. In doing so, the focus is not so much the discussion of turbulent burning velocity models but rather how to use these models in an engine code and to put forward a framework allowing them to be compared or evaluated. For convenience and availability of figures, work at Leeds or Ghent is frequently quoted rather than other, equivalent works of equal merit.

\section{Thermodynamic modelling}

As discussed in the introduction, the basis for multi-zone models is formed by consideration of conservation of mass and energy. In the following, the equations for the cylinder pressure and temperature(s) are derived. This will show where additional information, in the form of sub-models, is necessary in order to close these equations.

\subsection{Modelling assumptions}

Before conservation of energy is written out for the cylinder volume, from inlet valve closing time to exhaust valve opening time (i.e. the power cycle), some assumptions are generally adopted to simplify the equations. During compression and expansion, pressure is invariably assumed uniform throughout the cylinder, with fixed unburned and burned gas regions in chemical equilibrium (see Section 2.2.). During flame propagation, burned and unburned zones are assumed to be separated by an infinitely thin flame front, with no heat exchange between the two zones. All gases are considered ideal gases; possible invalidity of the ideal gas law at high pressures is countered by the associated high temperatures under engine combustion conditions. 


\subsection{Sub-models}

The derivation of the governing equations is given in Appendix A. Solving for the pressure and temperature during compression (Eqs. (A.4) and (A.6)) requires:

- $\quad$ Trapped conditions, i.e. the initial conditions at the start of compression, at intake valve closing (IVC) time; e.g. the pressure at IVC, the fresh mass of air and fuel (and EGR if applicable), the residual gas fraction and the equivalence ratio. The trapped conditions can result from calculation of the intake stroke (gas dynamics), a combination of measured variables and estimation, or from models (e.g. for the residual mass fraction $[6,7])$.

- The cylinder volume as a function of crank angle and the rate of change of cylinder volume. These are functions of the engine geometry: bore, stroke, connecting rod length and compression ratio.

- $\quad$ Gas properties $\left(c_{v}, R\right)$ : a number of sources exist to determine the cylinder gas properties as a function of temperature, e.g. the JANAF data tables [8], and others $[9,10]$.

- A blow-by model, to determine the mass leaving or entering the cylinder 'control volume' ( $d m_{l} / d \theta$ in the equations in appendix). Models of varying complexity have been proposed in the literature (e.g. [9]). Some research engines (particularly these with optical access) can have substantial blow-by rates and therefore require an accurate blow-by model. Even for engines with low blow-by rates, the mass trapped in the top land crevice can be considerable at certain times in the cycle. This mass may or may not burn and, if it burns, its combustion may occur late in the cycle, rather than during the main flame propagation event.

- $\quad$ A heat transfer model ( $d Q / d \theta$ in the equations in appendix). Again, a number of different models are in common use, of which the best known are those of Woschni [11] and Annand [12]. These use the cylinder bore as a 'characteristic length' and the mean piston speed as a 'characteristic speed' to determine a Reynolds number. If one wishes to use more relevant incylinder turbulence quantities such as the integral length scale and the rms turbulent velocity, an in-cylinder turbulence sub-model is required (see below).

Solving for the pressure, unburned gas temperature and burned gas temperature during combustion (Eqs. (A.21), (A.24) and (A.26)) necessitates a model for the mass burning rate $d m_{x} / d \theta$, in addition to the data and sub-models described above.

During combustion, the burned gas composition is generally calculated on the assumption of chemical equilibrium at the given temperature and pressure; typically, up to 12 combustion product species $\left(\mathrm{H}_{2} \mathrm{O}, \mathrm{H}_{2}, \mathrm{OH}, \mathrm{H}, \mathrm{N}_{2}, \mathrm{NO}, \mathrm{N}, \mathrm{CO}_{2}, \mathrm{CO}, \mathrm{O}_{2}, \mathrm{O}\right.$ and $\left.\mathrm{Ar}\right)$ are invoked. If interested in calculating the emissions, one also has to consider the chemical kinetically controlled reactions, such as those for NO. This is discussed in Section 4.

In summary, multi-zone engine modelling requires sub-models for:

- Blow-by

- Heat transfer 
- $\quad$ Burned gas composition

- Mass burning rate

- In-cylinder turbulence, as needed by the heat transfer and mass burning rate sub-models either based on experimentally derived data, or from cold flow CFD calculations (generally $k-\varepsilon$ type models, for a review see ref. [13].

The mass burning rate sub-model is treated in the next section.

\section{Mass burning rate sub-model}

To close the equations for the cylinder pressure, and temperature of burned and unburned zone, a mass burning rate model is needed. The features of such a model and the processes they try to represent are developed in the following.

\subsection{Ignition}

If one is primarily interested in the indicated work and efficiency, in other words simulation of the complete engine cycle, the ignition of the cylinder charge is usually not modelled in detail. In fact generally, the ignition is not modelled at all; but rather the start of combustion is initialised by assuming the instantaneous formation of an ignition kernel at or shortly after the ignition timing. The ignition kernel is often ascribed a certain mass or volume. Some examples are:

- $m_{b}=0.01 m_{\text {tot }}$, the initial flame kernel having a mass equal to one percent of the total cylinder mass $m_{t o t}$;

- $\quad m_{b}=0.02 m_{t o t}$, as adopted by Wu et al. [14] (after a modelled 'ignition delay time');

- $\quad 2 r_{f}=1 \mathrm{~mm}$, with $r_{f}$ the flame kernel radius, as assumed by Brehob and Newman [15] and Verhelst and Sierens [16];

- $\quad 2 r_{f}=$ electrode gap [17];

- $\quad V_{b}=V_{c y l} / 1000$, with $V_{b}$ the volume of the initial flame kernel and $V_{c y l}$ the maximum cylinder volume (swept+clearance), after Benson et al. [18];

- If the engine is optically accessible, one can initiate an ignition kernel on the basis of some observed natural light or schlieren image derived mean flame radius at a given crank angle [19]

As the ignition process is dependent on local parameters around the spark plug [20] and thermodynamic modelling, by its nature, uses mostly (or exclusively) global (bulk) parameters, such initialisation is logical, even though it is quite arbitrary. When the ignition is not critical (stoichiometric mixture, at moderate engine speeds), this is probably justified. At conditions that are sensitive to ignition (lean mixtures, high engine speeds), such simplifications may be less justifiable. 


\subsection{Initial phase of combustion}

Pictures of the initial phase of combustion show an initially quasi-spherical, relatively smooth flame kernel. Thus, one can assume the initial combustion to proceed in a quasi-laminar fashion, with the mass burning rate $\dot{m}_{b}$ given by:

$$
\dot{m}_{b}=\rho_{u} A u_{n r}
$$

Here, $\rho_{u}$ is the unburned gas density, $A$ is the flame area defined at the cold flame front, and $u_{n r}$ is the stretched laminar burning velocity based on the rate of production of reacted gas [21].

When the flame kernel becomes larger than the smallest turbulent eddies, the flame front will become wrinkled. As the kernel grows its flame front will become gradually more wrinkled as it experiences a growing spectrum of turbulent length scales. This can be observed in Fig. 2, which shows successive laser sheet (mie scatter) flame images, superimposed on the in-cylinder velocity field which is depicted by manually tracked streaks using particle tracking velocimetry (PTV). These images may be thought of as two-dimensional 'slices' through three-dimensional flame structures such as that shown in Fig. 1. The figure shows a period from $20.25^{\circ} \mathrm{ca}$ bTDC to $6.75^{\circ} \mathrm{Ca}$ aTDC and was taken from experiments using a research engine with full bore overhead optical access, under "quiescent" (no bulk flow) conditions. Details can be found in the paper by Cairns and Sheppard [22]. However, if one takes into account the (practical) choice for the initialisation of combustion in the engine model, see above, a problem arises. Using the assumption $m_{b}=0.01 m_{t o t}$, the resulting flame kernel radius is of the order of $10 \mathrm{~mm}$. Using the assumption $V_{b}=V_{c y l} / 1000$, the flame kernel radius is of the order of $2 \mathrm{~mm}$. This has to be compared to the length scale of the smallest turbulent eddies, the Kolmogorov length scale $l_{K}$, which depends on the engine operating conditions and can be roughly estimated to be between $3 \mu \mathrm{m}$ and $0.6 \mathrm{~mm}$, according to Abraham et al. [23]. It is clear that this means that, for the calculations, there is never a truly laminar phase: after the computational initialisation of combustion, the resulting flame kernel will conceptually be instantaneously subjected to a portion of the turbulent spectrum. In some cases, e.g. with the assumptions concerning the burned mass, the kernel will be larger than the integral length scale (estimated to be between 1.5 and $3 \mathrm{~mm}$ according to Abraham et al. [23]). Such assumptions clearly skip a large part of the initial combustion phase.

One can question the necessity of a flame development phase (with the flame becoming increasingly wrinkled by a growing spectrum of wrinkling length scales) in engine modelling, if part or all of this phase is effectively skipped because of the choice of initialisation of combustion. Nevertheless, numerous papers report the necessity of including such a term, as using a 'fully developed' turbulent burning velocity (see later) results in an underestimation of, for example, the duration of the $0-10 \%$ mass fraction burned phase. The flame diameter at initialisation of combustion is at least an order of magnitude smaller than the largest length scales so there is still a substantial flame development phase. 
By analogy with the laminar case, a turbulent burning velocity can be defined as:

$$
u_{t(e / r)}=\frac{\dot{m}_{(e / r)}}{\rho_{u} A}
$$

Where the distinction has been made between a turbulent 'engulfment' velocity $u_{t e}$ and a turbulent 'reacted' burning velocity $u_{t r}$ based on the rate of production of burned gas [4]. The flame front is now a wrinkled one and thus, the choice of the relevant flame area $A$ is a lot less straightforward than in the laminar case. Even in well controlled experiments, e.g. in constant volume combustion bombs, care is needed when picking a flame area $[4,21]$. It goes without saying that selecting one in engine combustion is a lot more difficult.

The most frequently used approach for engine combustion models is to model the flame area by a spherical flame front truncated by the cylinder walls and the piston, centred at the spark plug (in some models, the centre can be moved by bulk flow motions [24]). Experiments have shown that the spherical geometry is a good approximation, certainly for "quiescent" conditions, see Fig. 2). In thermodynamic modelling, this is also the most logical a priori choice. Deformation of the flame by the in-cylinder flow would need detailed information on the flow motions, which is contradictory to the thermodynamic modelling approach.

Thus, the modelling challenge shifts to constructing an appropriate model of an equivalent turbulent burning velocity. It is very important that the definition for $A$ and the model for $u_{t}$ are compatible. For instance, if $A$ is viewed as a surface that completely encloses the real turbulent flame front, $u_{t}$ is an 'engulfment' velocity, $u_{t e}$. With this choice, combustion will not be completed even when $A$ is such that the complete cylinder volume has become contained within its boundary. A model for the burn-up of the remaining unburned charge then needs to be invoked (see later).

As stated previously, during the flame development phase, the flame front is not yet wrinkled by the full turbulent spectrum. As most models for the turbulent burning velocity have been derived for fully developed flames, a sub-model is needed to account for this effect on $u_{t}$. Again, one has to be careful when selecting such a sub-model from the literature. This is explained in the following.

First, consider some formulations of flame development sub-models:

- Abdel-Gayed et al. [25] measured turbulent velocities and integral length and time scales in a fanstirred bomb (without combustion) using laser Doppler velocimetry (LDV) to obtain the turbulence power spectral density function. They defined an effective rms turbulent velocity $u_{k}^{\prime}$ (effective as in effectively enhancing burning rates) and related it to the rms turbulent velocity $u^{\prime}$ through $\left(u_{k}^{\prime}\right)^{2}=u^{\prime 2} f(P S D)$ where $f(P S D)$ is the power spectral density function (PSD) integrated from time zero (time of ignition) to the time elapsed since flame initiation. A best fit through $u_{t k} / u_{t}$ versus dimensionless time, where $u_{t k}$ is the developing turbulent burning velocity and $u_{t}$ 
is the developed turbulent burning velocity, yielded:

$\frac{u_{t k}}{u_{t}}=\left[1-e^{-0.2 t_{k} / \tau_{a}}\right]^{0.75}$

here, $t_{k}$ is the time elapsed from ignition and $\tau_{a}$ is a time constant given by $l_{I} / u^{\prime}$ with $l_{I}$ the integral length scale.

- Keck et al. [26] reported schlieren and pressure measurements in an optical engine and fitted an entrainment model (see later) to the results. They added a term of the form $1-\exp -t / \tau$ to the entrainment rate to improve the correspondence between measurement and simulation for the initial flame growth, explaining that significant wrinkling of the flame front only occurs after the flame has burned from the spark plug to the edge of the turbulent eddy in which the spark occurred. Brehob and Newman [15] replaced $t / \tau$ with $r_{f} / r_{c}$, where $r_{f}$ is the flame radius and $r_{c}$ is a 'critical' radius of the order of the integral length scale. The replacement was cited to yield better representation of length scales that effectively wrinkle the flame. Later, an additional term appeared in the 'GESIM' entrainment model formulation by Dai et al. [27], of the form $r_{f} / r_{c}{ }^{1 / 3}$, again for a better correspondence between measurement and simulation at the start of combustion. Dai et al. [27] cited Brehob and Newman 15] for this formulation, although no such term is reported in that paper.

These terms clearly represent the flame development, through a multiplying factor:

$$
1-e^{-r_{f} / r_{c}}\left(\frac{r_{f}}{r_{c}}\right)^{1 / 3}
$$

- In an adaptation of an earlier formulation by Matthews and Chin [17] of a fractal combustion model, Wu et al. [14] replaced the rms turbulent velocity $u^{\prime}$ used in their model, with a $u_{\max }$ to express the flame wrinkling by an increasing range of turbulent length scales during flame growth. The velocity $u_{\max }$ is the eddy velocity associated with the largest eddies that can wrinkle the flame, their size is assumed to be the flame radius $r_{f}$, with $u_{\max }$ determined from energy cascade arguments:

$\varepsilon=\frac{u^{\prime 3}}{l_{I}}=\frac{u_{\max }^{3}}{r_{f}}$ where $\varepsilon$ is the rate of dissipation of turbulent kinetic energy, thus:

$u_{\max }=u^{\prime}\left(\frac{r_{f}}{l_{I}}\right)^{1 / 3}$

resulting in a term similar to the second term in Eq. (1.4).

- Lipatnikov and Chomiak $[28,29]$ proposed an approximation of turbulent burning velocity development following Zimont's ideas ([30] and references cited therein), starting from a developing flame thickness controlled by a transient turbulent diffusivity. The resulting relation 
between developing and developed turbulent burning velocity is as follows:

$$
\frac{u_{t, t}}{u_{t}}=\left\{1+\frac{\tau^{\prime}}{t^{\prime}}\left[\exp \left(-\frac{t^{\prime}}{\tau^{\prime}}\right)-1\right]\right\}^{1 / 2}
$$

where $u_{t, t}$ is the transient (developing) turbulent burning velocity, $t^{\prime}$ is the time from ignition and the time constant $\tau^{\prime}$ is given by $D_{t} / u^{\prime 2}$, with $D_{t}$ the developed turbulent diffusivity. Using the predictions and constants of the $k-\varepsilon$ turbulence model used in ref.[28], $\tau^{\prime}$ is given by $0.55 l_{I} / u^{\prime}$.

- Morel et al. [24] use a multiplying factor for $u^{\prime}$ of the form:

$$
1-\frac{1}{1+C\left(\frac{r_{f}}{l_{I}}\right)^{2}}
$$

with $C$ a calibration constant.

This list of flame development sub-models is not exhaustive and merely serves to illustrate the following points.

Equations (1.4) and (1.5) lead to infinity when $r_{f} \rightarrow \infty$, which is not physically correct, they can therefore be considered as inferior to Eqs. (1.3), (1.6) and (1.7) which have an asymptotical value of unity. Equations (1.3) and (1.6) have been compared by Lipatnikov and Chomiak [29] and are quite similar. Both are implemented in a straightforward manner in an engine code, as they are multiplying factors, or 'flame development factors' - FDF, giving the 'developing' turbulent burning velocity from the 'developed' turbulent burning velocity as given by the turbulent burning velocity model:

$u_{t, t}=F D F \cdot u_{t}$

Hence, they are easily used as a separate sub-model, or building block, of the mass burning rate model. This is not the case with the formulations of Eqs. (1.4), (1.5) and (1.7), used as multiplying factors of $u^{\prime}$ in the original works.

\subsection{Fully developed turbulent combustion}

Once the flame has grown sufficiently large for its surface to be wrinkled by the complete spectrum of turbulent length scales, conceptually the phase of 'fully developed' turbulent flame propagation starts. There is still uncertainty about the parameters controlling the turbulent burning velocity. Although the influences of rms turbulent velocity and laminar burning velocity have been cited in numerous papers, the effects of the turbulent length scales, pressure and mixture diffusivities are less clear (for an excellent review, see ref. [29]). However, it is obvious that in engines the turbulent burning velocity constantly changes; as pressure and temperature changes (influencing the laminar burning velocity among others). Thus, 'fully developed' turbulent flame propagation should not be confused with a constant burning velocity or flame brush thickness $\delta_{t}$. As described above, and evident from Fig. 2, 
the turbulent flame brush thickness initially increases as the flame grows. It is difficult to measure or estimate the flame brush thickness evolution after the initial combustion phase, because of the complications of end-gas compression and piston induced expansion; with corresponding changes in pressure and temperature, which could increase as well as decrease $\delta_{t}$ [31].

The corresponding phenomenology at the end of combustion will be considered first, before discussing ways of modelling the above.

\subsection{End of combustion}

As the flame approaches the walls, the spectrum of turbulent scales able to wrinkle the flame decreases and the mass burning rate decreases. Increased heat losses from the flame, due to its proximity to the walls, also decreases the mass burning rate. Finally, the flame extinguishes due to exhaustion of reactants, excessive heat loss or radical termination at the walls. The final mass burning rate is well approximated by an exponential decay [32].

From the foregoing it is clear that the turbulent burning velocity parameter by itself is insufficient to describe a turbulent flame in an engine. Such a flame is confined by the chamber walls, thus for an accurate description of the end of combustion it is prerequisite to include information on the flame brush thickness.

\subsection{A modelling framework}

If the burned mass fraction $m_{b}$ is plotted versus crank angle, the well-known $S$ shaped curve results (see Fig. 3). This arises from the phenomena described above, of a slow start of combustion, gradually increasing in rate as the flame develops, a 'fully developed' phase and a slowing rate of combustion as the flame approaches the walls.

Blizard and Keck [33] postulated a combustion mechanism comprising entrainment into the flame front, with a velocity $u_{e}$, of turbulent eddies of characteristic size $l_{e}$. These eddies were presumed to burn inwards from peripheral ignition sites to be consumed in a time $\tau \sim l_{e} / u_{l}$; with $u_{l}$ the laminar burning velocity. This can be described by the following set of equations:

$$
\begin{aligned}
& \dot{m}_{e}=\rho_{u} A_{f} u_{e} \\
& \dot{m}_{b}=\frac{m_{e}-m_{b}}{\tau}
\end{aligned}
$$

With $m_{e}$ is the mass entrained (engulfed) behind the mean leading edge of the flame front, $A_{f}$ a flame surface area, $u_{e}$ a velocity and $\tau$ a time constant. This set of equations is known as the entrainment equations, entrainment combustion model, or eddy burning model. Shown in Fig. 3 is the resultant behaviour in mass fraction burned $\left(m_{b}\right)$, as combustion slowly develops during a few time 
constants $\tau$, lagging behind $m_{e}$ during the 'fully developed' phase and then slowly approaching the total cylinder mass towards the end of the combustion event.

Entrainment combustion models thus assume the combustion to take place in two steps: first, unburned mass is entrained by the flame front with a rate given by Eq. (1.9). Then, the entrained turbulent eddies burn in a time that is a function of the eddy size and the laminar burning velocity, so the mass burning rate is given by Eq. (1.10). Equations (1.9) and (1.10) are the basis of many combustion models, with differences primarily arising from the choice of the characteristic eddy size ( $l_{e}$, leading to different corresponding values for $\tau$ ) and entrainment velocity $\left(u_{e}\right)$. Blizard and Keck [33] fitted their model to experiments and scaled $l_{e}$ with intake valve lift and $u_{e}$ with intake jet gas speed. Later, Keck [32] further adapted the model by adding a laminar term to the mass burning rate equation and taking the integral length scale $l_{I}$ as the characteristic eddy size [26].

Tabaczynski and co-workers [34-36] introduced the turbulence structure suggested by Tennekes into the entrainment framework. This led to a combustion mechanism where eddies of integral scale $l_{I}$ are entrained and ignition sites propagate along vortex tubes of size $l_{K}$ (the Kolmogorov length scale), of which the spacing is given by the Taylor length scale of turbulence, $l_{T}$. The Taylor length scale was assumed to be the characteristic size at which laminar diffusion becomes important. The entrainment velocity $u_{e}$ was taken to be $u^{\prime}+u_{l}$. The expression for the characteristic burning time of entrained eddies has been adapted through the years, Tabaczynski et al. [34] assumed it was the time for an eddy of the Taylor length scale to be consumed through laminar flame propagation, $\tau \sim l_{T} / u_{l}$. Tabaczynski's model is still the basis of today's 'GESIM', Ford's 'general engine simulation' code, with a number of adaptations accounting for flame stretch, flame development, etc. [27] (see below).

Blizard and Keck [33] derived the entrainment equations from a proposed hypothesis of how flame propagation takes place in an engine. Tabaczynski et al. $[34,36]$ use the same premise with additional assumptions on the nature of turbulence in engines. It must be emphasized that, in consequence, the entrainment equations were originally never a phenomenological model of flame propagation but rather a mathematical description that agreed with the S-shaped mass fraction burned observation. The reality of an entrainment type combustion mechanism is quite uncertain and has never been experimentally observed. Experiments indicate a continuous flame front, which has been shown to be able to fold onto itself in certain cases creating pockets of unburned gas that burn up inwards [31]. Thus, the combustion in engines falls in the flamelet regime in most cases (also termed the reaction sheet regime or wrinkled laminar flames regime), with the turbulence influencing the flame through wrinkling and straining, but not significantly altering the transport processes in the reaction zone. This greatly simplifies modelling, as chemical effects can be subsumed into a laminar burning velocity. 
It is also important to note that, as a approximation of the observed S-shape, Eqs. (1.9) and (1.10) lead to a flame development phase due to $m_{b}$ 'developing' during a few time constants $\tau$. As most models using the entrainment equations also incorporate a flame development sub-model, it should be pointed out that the simulated flame development is influenced both by the form of the equations as well as the sub-model.

As stated above, flame propagation within an engine may be characterised by a turbulent burning velocity (with the associated flame surface area) and a flame brush thickness. In the framework constituted by Eqs. (1.9)and (1.10), the turbulent burning velocity and associated flame surface are represented by $A_{f}$ and $u_{e}$ in Eq. (1.9), the flame brush thickness is represented by the time constant $\tau$ in Eq. (1.10). This is how Eqs. (1.9) and (1.10) should best be interpreted: as a mathematical representation of turbulent flame propagation inside an engine, with allowance for the effects of a finite flame thickness $\delta_{t}$. This viewpoint does not follow the 'historical' distinction in engine modelling literature between 'eddy-burning' and 'flamelet' models (made e.g. by Heywood [37]). It is perfectly possible (and reasonable) to evaluate a model for the turbulent burning velocity based on flamelet regime assumptions by using the $u_{t}$ model to provide values for $u_{e}$ in Eq. (1.9), provided the appropriate choices are made for $A_{f}$ and $\tau$. Engine combustion models directly modelling $\dot{m}_{b} \sim \rho_{u} A_{f} u_{t r}$ have been reported to need special measures for a correct simulation of the end of combustion [14]. This comes as no surprise in the light of the above: the effects of the flame brush thickness need to be allowed for in some way. Bozza et al. [38] use $\dot{m}_{b} \sim \rho_{u} A_{f} u_{t r}$ (with a fractal combustion model for $u_{t}$ ) until the flame diameter plus the integral length scale equal the cylinder bore. When this happens, the overall burning rate is taken to be a weighted mean of $\rho_{u} A_{f} u_{t r}$ and a 'wall-combustion burning rate', given by an equation similar to Eq. (1.10). Thus, they assume a flame brush thickness equal to the integral length scale. This approach has the advantage of decoupling the modelling of the flame development phase (e.g. through a flame development factor) from the modelling of the end-of-combustion phase (although Bozza et al. [38] modify the fractal dimension used by the fractal combustion model to account for the flame development).

For disc chambers with central ignition, the assumption of a spherical flame leads to a (modelled) approach to the wall that occurs instantaneously around the circumference of the cylinder. Clearly, in reality this never happens: real flames are not spheres, and the flame centroid mostly drifts from the geometric centre of the chamber. Consequently, a calculated flame area, that discontinuously drops to zero when the flame reaches the wall leads to an incorrect simulation of the end of combustion. One could use a 'de-developing' turbulent burning velocity, based on one of the flame development models discussed in Section 3.2, when the distance between the flame and the wall becomes less than some maximum eddy size (i.e. the integral length scale [19]). The distance between flame and wall would 
then be the governing parameter instead of the flame radius (or rather, the ratio of the distance to the integral length scale).

For modern, asymmetric pent roof chambers, this is not such a problem as the flame approach to the wall is inevitably non-uniform.

\subsection{Laminar burning velocity}

Nearly all turbulent burning velocity models, as well as many CFD formulations, require laminar burning velocity data for the air/fuel/residual mixture at the instantaneous in-cylinder pressure and unburned gas temperature. Models that assume the local burning to take place in a laminar fashion (flamelet type models), need data on the stretched laminar burning velocity. This implies the need for either a library of stretched flamelets or a model for the effect of stretch rate.

A number of stretch models for use in turbulent combustion modelling have been suggested, most of which embody the effects of stretch rate in a factor $I_{0}$, with $u_{n}=I_{0} u_{l}$ [29]. Most of these models assume a linear relation between flame speed and stretch rate, valid for weakly perturbed laminar flames. Models for $I_{0}$ have been proposed, tailored for use in spark ignition engine modelling $[14,20,27]$.

However, calculating the local flame speed from stretch-free data and a stretch model requires stretchfree data, naturally. As of today, there is insufficient data on stretch-free burning velocities at engine conditions, for any fuel. Stretch and instabilities hamper the experimental determination of stretch-free data at higher (engine-like) pressures [39]. Recent work by Bradley et al. [40] shows that accounting for the effects of stretch at higher pressures is possible, but involves cumbersome experiments and large uncertainties, especially in relation to the determination of the Markstein numbers (a measure of the sensitivity of the flame to stretch rate).

Most of the currently used correlations for laminar burning velocity were derived from the pressure development recorded in a constant volume combustion bomb, e.g. the seminal work and widely adopted correlation for iso-octane/air of Metghalchi and Keck [41]. However, it should be noted that their correlations are not of stretch-free burning velocities $u_{l}$ but include (mostly unknown) effects of stretch and combustion instabilities. Additionally, since such correlations already encompass stretch effects it is perhaps inappropriate to use them together with a stretch factor $I_{0}$, as done in some modelling studies. It has been suggested that at the relatively large flame radii at which high pressure/temperature laminar flame speeds have been calculated for bomb experiments, stretch rate is small and hence its effect would be negligible. Unfortunately, it is precisely at such low stretch conditions that high pressure flames are most prone to instabilities such as cellularity, which have been shown to have even more marked effect on burning velocity than stretch rate [21]. The extent to which cellular enhancement of 'laminar' burning velocity carries over to turbulent flames has not yet been resolved.

It is perhaps remarkable that engine models making use of such correlations can give reasonably accurate results. This may be associated with the fact that they generally employ experimentally derived relationships for turbulent burning velocity expressed as functions of the ratio of measured 
turbulent velocity to corresponding laminar burning velocity at the same pressure and temperature. This perhaps suggests that instability induced enhancement of laminar burning velocity does carry over to turbulent flames to a certain extent.

For the time being, it is perhaps best to consider $u_{l}$ as a characteristic chemical reaction rate (as used to determine the burn-up time constant in Eq. (1.10)) and as a correlating parameter for $u_{t}$ in experimentally derived expressions.

\section{Emissions}

When one is primarily interested in the flame propagation and resulting pressure development, one can assume the burned gas composition to approximate that calculated from chemical equilibrium. However, some formation and destruction reactions are actually slow compared to the duration of the engine cycle and thus, for some components the engine-out emissions can deviate substantially from equilibrium concentrations. This is the case for $\mathrm{NO}$ and $\mathrm{CO}$ emissions. Some success has been reported for de-coupling of kinetically controlled $\mathrm{CO}$ oxidation and $\mathrm{NO}$ formation mechanisms from the main combustion reactions, with the latter remaining in thermochemical equilibrium. However, modelling of engine emissions is not within the scope of this paper, the interested reader is referred elsewhere for models that can be integrated in the multi-zone thermodynamic modelling framework $[9,42]$.

\section{Abnormal combustion phenomena}

As noted in the Introduction, multi-zone thermodynamic models are quite widely adopted for parametric investigation of new combustion concepts/designs. These often encompass operating conditions (e.g. highly turbocharged, high exhaust gas recirculation) which are susceptible to end gas autoignition induced knock. Such models have therefore been adapted for prediction of autoignition (and so knock) onset by using computed end gas pressure/temperature history to drive autoignition models ranging from simple empirical single step chemistry routines [43] through the well known "Shell" scheme [44] to quite complex chemical reaction schemes [45]; sometimes incorporating feedback of heat release associated with the early stages of autoignition development back into calculation of the in-cylinder pressure and unburned gas temperature. It has been suggested that correct prediction of the normal flame propagation is, via its control of the thermodynamic state of the end gas, the determinant of the accuracy of autoignition onset assessment - such that perhaps only use of the simplest autoignition schemes is currently justified [46], particularly given uncertainties in the detailed chemical mechanisms and associated reaction rate constants for real hydrocarbon fuel mixtures. However, detailed consideration of autoignition modelling is again outside the remit of the current work.

\section{Conclusions}

Intelligently used, multi-zone thermodynamic models can provide a valuable tool for the understanding and development of combustion in spark-ignition engines. To do this, they should reflect the essential chemistry and physics; noting their limitations (assumptions such as spherical flame propagation, 
spatial and temporal variation of turbulence, mixture homogeneity, wall conditions etc.). Given the level of the modelling assumptions necessary, it is clear that many alternative formulations of these models are essentially equivalent. However, because of the number of sub-models that are needed to provide a description of all relevant processes, it is quite easy to combine models that use contradictory assumptions (e.g. using different flame areas, mixing $\mathrm{u}_{\mathrm{te}}$ 's with $\mathrm{u}_{\mathrm{tr}}{ }^{\prime} \mathrm{s}, \ldots$ ). Some of these issues are addressed in the current overview:

- Depending on the method of initialising the calculation of the mass burning rate, a significant part of the flame development phase is effectively skipped. This could compromise the predictive capability of the engine model, when examining the effects of settings that affect the in-cylinder turbulence, e.g. for other engine speeds, tumble flow valve settings, ...

- Several flame development models have been used in the literature. The ones expressing the developing turbulent burning velocity as a factor of the 'fully-developed' turbulent burning velocity are most easily integrated in an engine code (decoupling of sub-models).

- The turbulent burning velocity is only one of the parameters required for modelling the flame propagation inside engines; the associated turbulent flame brush thickness also needs to be described in one way or another. The 'entrainment' equation set is a mathematical representation that provides a way of modelling the turbulent burning velocity with incorporation of the effects of the turbulent flame thickness.

- From the discussion on the laminar burning velocity $\mathrm{u}_{\mathrm{l}}$, it can be concluded that a number of fundamental questions concerning $u_{l}$, e.g. the effects of instability, and how this carries over to $u_{t}$, remain unresolved. This also increases the motivation for the use of multi-zone thermodynamic models as a framework in which hypotheses concerning the nature of flame propagation in engines can be easily tested.

Hopefully, this overview has helped to clarify the way in which all sub-models are linked and helps to set a framework in which different sub-models can be compared on the same basis.

\section{Acknowledgements}

Fruitful discussions with colleagues at Ghent and Leeds (D. Bradley, A. Burluka, M. Lawes, R. Sierens) are gratefully acknowledged. Thanks also to Dr. R. Woolley, University of Sheffield, for his contribution to various Leeds experiments which have led to most of the pictures shown here; to the Rapid Prototyping team at the School of Mechanical Engineering in Leeds for making the 3D reconstruction shown in Fig. 1; and to many postgraduate students at Ghent and Leeds for their contributions to experiments and simulations.

\section{Appendix A - quasi-dimensional model equations}

The basic equation for the engine model is derived from the conservation of energy applied to the cylinder volume:

$$
d U=-\delta Q-\delta W+\sum_{i} h_{i} d m_{i}
$$


Here, $U$ is the internal energy of the cylinder gas mixture, $Q$ the heat exchange of the cylinder contents with the environment (walls) where $Q>0$ for heat loss from gas to wall, $W$ the work on the piston where $W>0$ for work delivered by the cylinder charge, $h_{i}$ the specific enthalpy of in- or outflowing gas, and $d m_{i}$ the mass flow into (+) or out of (-) the cylinder. The work $\delta W$ can be expressed as $p d V$, where $p$ is the pressure and $V$ the cylinder volume.

The first term on the right hand side of Eq. (A.1) expresses the heat loss of the cylinder contents to the surroundings, the modelling of which is described in Section 2.2. The second term expresses the work delivered, the third term is the total energy flowing into or out of the cylinder. Here, only the power cycle is considered so the change in cylinder mass is solely through blow-by. During the power cycle only leakage from the cylinder volume to the crankcase is assumed (no inflow) and the blow-by composition is taken to be the cylinder gas composition. However, a reverse flow, from crevices to the combustion chamber, is possible during the expansion stroke when, due to piston motion, the crevice pressure is higher than the cylinder pressure. This is usually included in the blow-by model. If a reverse flow occurs, one also needs to consider the burn-up of the returning gases.

In the following sections Eq. (A.1) is developed for the cases compression/expansion and combustion. Here, this is done on a mass basis but the equations can also be cast in molar form.

\section{A.1. Compression and expansion}

Starting from conservation of energy, with the change in cylinder mass solely from blowby:

$$
\frac{d(m u)}{d \theta}=-\frac{d Q}{d \theta}-p \frac{d V}{d \theta}+h \frac{d m}{d \theta}
$$

Where the left hand side can be written as:

$$
m \frac{d u}{d \theta}+u \frac{d m}{d \theta}
$$

With $d u / d \theta=\partial u / \partial T \cdot d T / d \theta=c_{v} d T / d \theta$. For an ideal gas, $h=u+R T$, where $R$ is the mixture gas constant, resulting in the following equation for the temperature change:

$$
\frac{d T}{d \theta}=\frac{1}{m c_{v}}\left[-\frac{d Q}{d \theta}-p \frac{d V}{d \theta}+\frac{d m}{d \theta} R T\right]
$$

With $d m / d \theta$ resulting from blow-by: $d m / d \theta=d m_{l} / d \theta$. For the pressure change, the ideal gas equation $p V=m R T$ is differentiated: 


$$
V \frac{d p}{d \theta}+p \frac{d V}{d \theta}=R T \frac{d m}{d \theta}+m T \frac{d R}{d \theta}+m R \frac{d T}{d \theta}
$$

During compression, the cylinder gas composition can be assumed constant, during expansion it can be assumed to change only slowly, or $d R / d \theta \approx 0$, resulting in the following equation for the pressure change:

$$
\frac{d p}{d \theta}=\frac{1}{V}\left(\frac{d m_{l}}{d \theta} R T+m R \frac{d T}{d \theta}-p \frac{d V}{d \theta}\right)
$$

\section{A.2. Combustion}

Conservation of energy applied to the unburned gas zone results in the following equation:

$$
\frac{d m_{u} u_{u}}{d \theta}=-\frac{d Q_{u}}{d \theta}-p \frac{d V_{u}}{d \theta}-h_{u} \frac{d m_{x}}{d \theta}-h_{u} \frac{d m_{l, u}}{d \theta}
$$

here, $Q_{u}$ is the heat exchange between the unburned zone and the cylinder walls, $V_{u}$ is the volume of the unburned zone, $d m_{x} / d \theta$ is the mass burning rate and $d m_{l, u} / d \theta$ is the leakage of unburned gas from cylinder to crankcase $\left(d m_{l, u} / d \theta>0\right)$. Again, the left hand side can be written as:

$$
m_{u} \frac{d u_{u}}{d \theta}+u_{u} \frac{d m_{u}}{d \theta}
$$

With $d u_{u} / d \theta=c_{v, u} d T_{u} / d \theta$. The rate of change in the unburned gas can be written as:

$$
\frac{d m_{u}}{d \theta}=-\frac{d m_{x}}{d \theta}-\frac{d m_{l, u}}{d \theta}
$$

Resulting in:

$$
\begin{aligned}
& m_{u} c_{v, u} \frac{d T_{u}}{d \theta}-u_{u} \frac{d m_{x}}{d \theta} \\
& =-\frac{d Q_{u}}{d \theta}-p \frac{d V_{u}}{d \theta}-h_{u} \frac{d m_{x}}{d \theta}-R_{u} T_{u} \frac{d m_{l, u}}{d \theta}
\end{aligned}
$$

Conservation of energy for the burned gas zone results in: 


$$
\frac{d m_{b} u_{b}}{d \theta}=-\frac{d Q_{b}}{d \theta}-p \frac{d V_{b}}{d \theta}+h_{u} \frac{d m_{x}}{d \theta}-h_{b} \frac{d m_{l, b}}{d \theta}
$$

Again,

$$
\frac{d m_{b} u_{b}}{d \theta}=m_{b} \frac{d u_{b}}{d \theta}+u_{b} \frac{d m_{b}}{d \theta}
$$

With $d u_{b} / d \theta=c_{v, b} d T_{b} / d \theta$. The rate of change in the burned mass can be written as:

$$
\frac{d m_{b}}{d \theta}=\frac{d m_{x}}{d \theta}-\frac{d m_{l, b}}{d \theta}
$$

Resulting in:

$$
\begin{aligned}
& m_{b} c_{v, b} \frac{d T_{b}}{d \theta}+u_{b} \frac{d m_{x}}{d \theta} \\
& =-\frac{d Q_{b}}{d \theta}-p \frac{d V_{b}}{d \theta}+h_{u} \frac{d m_{x}}{d \theta}-R_{b} T_{b} \frac{d m_{l, b}}{d \theta}
\end{aligned}
$$

Next the total internal energy balance is written out, as the sum of the balances (A.10) and (A.14):

$$
\begin{aligned}
& m_{u} c_{v, u} \frac{d T_{u}}{d \theta}+m_{b} c_{v, b} \frac{d T_{b}}{d \theta}+u_{b}-u_{u} \frac{d m_{x}}{d \theta} \\
& =-\frac{d Q}{d \theta}-p \frac{d V}{d \theta}-R_{u} T_{u} \frac{d m_{l, u}}{d \theta}-R_{b} T_{b} \frac{d m_{l, b}}{d \theta}
\end{aligned}
$$

Using

$$
\begin{aligned}
& \frac{d V}{d \theta}=\frac{d V_{u}}{d \theta}+\frac{d V_{b}}{d \theta} \\
& \frac{d Q}{d \theta}=\frac{d Q_{u}}{d \theta}+\frac{d Q_{b}}{d \theta}
\end{aligned}
$$

Differentiating the ideal gas equation for the two zones leads to:

$$
p \frac{d V_{u}}{d \theta}+V_{u} \frac{d p}{d \theta}=R_{u} T_{u} \frac{d m_{u}}{d \theta}+m_{u} R_{u} \frac{d T_{u}}{d \theta}
$$




$$
p \frac{d V_{b}}{d \theta}+V_{b} \frac{d p}{d \theta}=R_{b} T_{b} \frac{d m_{b}}{d \theta}+m_{b} R_{b} \frac{d T_{b}}{d \theta}
$$

If Eq. (A.18) is used to substitute $p d V_{u} / d \theta$ in Eq. (A.10), one obtains:

$$
\begin{aligned}
& m_{u} c_{v, u} \frac{d T_{u}}{d \theta}-h_{u}-R_{u} T_{u} \frac{d m_{x}}{d \theta}=-\frac{d Q_{u}}{d \theta}-V_{u} \frac{d p}{d \theta} \\
& -R_{u} T_{u} \frac{d m_{u}}{d \theta}-m_{u} R_{u} \frac{d T_{u}}{d \theta}-h_{u} \frac{d m_{x}}{d \theta}-R_{u} T_{u} \frac{d m_{l, u}}{d \theta}
\end{aligned}
$$

Using $c_{v, u}+R_{u}=c_{p, u}$ and Eq. (A.9), the following equation is obtained for the rate of change of the unburned gas temperature:

$$
\frac{d T_{u}}{d \theta}=\frac{1}{m_{u} c_{p, u}}\left(V_{u} \frac{d p}{d \theta}-\frac{d Q_{u}}{d \theta}\right)
$$

Now, substituting $d V_{u} / d \theta$ and $d V_{b} / d \theta$ in Eq. (A.16) using Eqs. (A.18) and (A.19), gives:

$$
\begin{aligned}
& \frac{d V}{d \theta}=\frac{R_{u} T_{u}}{p} \frac{d m_{u}}{d \theta}+\frac{m_{u} R_{u}}{p} \frac{d T_{u}}{d \theta}-\frac{V_{u}}{p} \frac{d p}{d \theta} \\
& +\frac{R_{b} T_{b}}{p} \frac{d m_{b}}{d \theta}+\frac{m_{b} R_{b}}{p} \frac{d T_{b}}{d \theta}-\frac{V_{b}}{p} \frac{d p}{d \theta}
\end{aligned}
$$

Using the ideal gas equation and Eqs. (A.9) and (A.13), this can be rewritten as:

$$
\begin{aligned}
& \frac{d V}{d \theta}=\left(\frac{V_{b}}{m_{b}}-\frac{V_{u}}{m_{u}}\right) \frac{d m_{x}}{d \theta}-\frac{V_{u}}{m_{u}} \frac{d m_{l, u}}{d \theta}-\frac{V_{b}}{m_{b}} \frac{d m_{l, b}}{d \theta} \\
& +\frac{V_{u}}{T_{u}} \frac{d T_{u}}{d \theta}+\frac{V_{b}}{T_{b}} \frac{d T_{b}}{d \theta}-\frac{V}{p} \frac{d p}{d \theta}
\end{aligned}
$$

Rearranging this equation to obtain an equation for the rate of change of the burned gas temperature:

$$
\frac{d T_{b}}{d \theta}=\frac{p}{m_{b} R_{b}}\left[\begin{array}{l}
\frac{d V}{d \theta}-\left(\frac{V_{b}}{m_{b}}-\frac{V_{u}}{m_{u}}\right) \frac{d m_{x}}{d \theta}+\frac{V_{u}}{m_{u}} \frac{d m_{l, u}}{d \theta} \\
+\frac{V_{b}}{m_{b}} \frac{d m_{l, b}}{d \theta}+\frac{V}{p} \frac{d p}{d \theta}-\frac{V_{u}}{T_{u}} \frac{d T_{u}}{d \theta}
\end{array}\right]
$$


Finally, substituting Eqs. (A.21) and (A.24) in Eq. (A.15) results in:

$$
\begin{aligned}
& \frac{m_{u} c_{v, u}}{m_{u} c_{p, u}}\left(V_{u} \frac{d p}{d \theta}-\frac{d Q_{u}}{d \theta}\right) \\
& {\left[\begin{array}{l}
\frac{d V}{d \theta}-\left(\frac{V_{b}}{m_{b}}-\frac{V_{u}}{m_{u}}\right) \frac{d m_{x}}{d \theta} \\
\left.+\frac{m_{b} c_{v, b} p}{m_{b} R_{b}}\left[\begin{array}{l}
V_{u} \\
m_{u}
\end{array}\right] \begin{array}{l}
d m_{l, u} \\
+\frac{V}{p} \frac{V_{b}}{m_{b}} \frac{d m_{l, b}}{d \theta}
\end{array}\right] \frac{V_{u}}{m_{u} T_{u} c_{p, u}}\left(V_{u} \frac{d p}{d \theta}-\frac{d Q_{u}}{d \theta}\right)
\end{array}\right]} \\
& +u_{b}-u_{u} \frac{d m_{x}}{d \theta} \\
& =-\frac{d Q}{d \theta}-p \frac{d V}{d \theta}-R_{u} T_{u} \frac{d m_{l, u}}{d \theta}-R_{b} T_{b} \frac{d m_{l, b}}{d \theta}
\end{aligned}
$$

Rearranging this equation to obtain the rate of change of the cylinder pressure:

$$
\begin{aligned}
& \frac{d p}{d \theta}= \\
& \left(\begin{array}{l}
\left.\frac{c_{v, u}}{c_{p, u}}-\frac{c_{v, b}}{R_{b}} \frac{R_{u}}{c_{p, u}} V_{u}+\frac{c_{v, b}}{R_{b}} V\right)^{-1} \\
-\left(1+\frac{c_{v, b}}{R_{b}}\right) p \frac{d V}{d \theta}-c_{p, b} T_{b} \frac{d m_{l, b}}{d \theta} \\
-\frac{R_{u}}{R_{b}} c_{p, b} T_{u} \frac{d m_{l, u}}{d \theta}-\frac{d Q}{d \theta} \\
-\left[\begin{array}{l}
u_{b}-u_{u}-c_{v, b} \\
\left.\left(T_{b}-\frac{R_{u}}{R_{b}} T_{u}\right)\right] d m_{x} \\
+\left(\frac{c_{v, u}}{c_{p, u}}-\frac{c_{v, b}}{R_{b}} \frac{R_{u}}{c_{p, u}}\right)
\end{array}\right\}
\end{array}\right.
\end{aligned}
$$

\section{References}

[1] Lawes M, Sheppard CGW, Woolley R. Three dimensional mapping of turbulent flame fronts. Proc. 9th Int. Symp. on Applications of Laser Techniques to Fluid Mechanics (Lisbon), 1998.

[2] Abdi Aghdam E, Burluka AA, Hattrell T, Liu K, Sheppard CGW, Neumeister J et al. Study of cyclic variation in an si engine using quasi-dimensional combustion model. SAE Technical Paper No. 2007-01-0939, 2007.

[3] Ayala FA, Heywood JB. Lean SI engines: the role of combustion variability in defining lean limits. SAE Technical Paper No. 2007-24-0030, 2007. 
[4] Bradley D, Haq MZ., Hicks RA, Kitagawa T, Lawes M, Sheppard CGW et al. Turbulent burning velocity, burned gas distribution, and associated flame surface definition. Combust Flame 2003;133:415-30.

[5] Blumberg PN, Lavoie GA, Tabaczynski RJ. Phenomenological models for reciprocating internal combustion engines. Prog Energy Combust Sci 1979;5:123-67.

[6] Senecal PK, Xin J, Reitz RD. Predictions of residual gas fraction in IC engines. SAE Technical Paper No. 962052, 1996.

[7] Fox JW, Cheng WK, Heywood JB. A model for predicting residual gas fraction in spark-ignition engines. SAE Technical Paper No. 931025, 1993.

[8] NIST-JANAF. NIST-JANAF Thermochemical Tables, Fourth edition. Journal of Physical and Chemical Reference Data, Monograph 9, 1998.

[9] Heywood JB. Internal Combustion Engine Fundamentals. McGraw-Hill, 1988.

[10] Reid RC, Prausnitz JM, Poling BE. The properties of gases \& liquids. 4th edn. McGraw-Hill, 1988.

[11] Woschni G. Universally Applicable Equation for the Instantaneous Heat Transfer Coefficient in the Internal Combustion Engine. SAE Technical Paper No. 670931, 1967.

[12] Annand WJD. Heat Transfer in the Cylinders of Reciprocating Internal Combustion Engines. Proc Instn Mech Engrs 1963;177(36):973-90.

[13] Agarwal A, Filipi ZS, Assanis DN, Baker DM. Assessment of single- and two-zone turbulence formulations for quasi-dimensional modeling of spark-ignition engine combustion. Combust Sci and Tech 1998;136:13-39.

[14] Wu C-M, Roberts CE, Matthews RD, Hall MJ. Effects of engine speed on combustion in SI engines: comparisons of predictions of a fractal burning model with experimental data. SAE Technical Paper No. 932714, 1993.

[15] Brehob DD, Newman CE. Monte Carlo simulation of cycle by cycle variability. SAE Technical Paper No. 922165, 1992.

[16] Verhelst S, Sierens R. A quasi-dimensional model for the power cycle of a hydrogen fuelled ICE. Int J Hydrogen Energy 2007;32:3545-54.

[17] Matthews RD, Chin Y-W. A fractal-based SI engine model: comparisons of predictions with experimental data. SAE Technical Paper No. 910079, 1991.

[18] Benson RS, Annand WJD, Baruah PC. A simulation model including intake and exhaust systems for a single cylinder four-stroke cycle spark ignition engine. Int. J. mech. Sci. 1975;17:97-124.

[19] Merdjani S, Sheppard CGW. Gasoline engine cycle simulation using the Leeds turbulent burning velocity correlations. SAE Technical Paper No. 932640, 1993.

[20] Herweg R, Maly RR. A fundamental model for flame kernel formation in S.I. engines. SAE Technical Paper No. 922243, 1992.

[21] Gillespie L, Lawes M, Sheppard CGW, Woolley R. Aspects of laminar and turbulent burning velocity relevant to SI engines. SAE Trans. 109, J of Engines (Section 3), 13-33, Sept 2001, also published as SAE Paper 2000-01-0192 (2000), and in "Advances in Combustion", Eds, Oppenheim, A.K., Stodolsky, F SAE SP-1492, ISBN 0-7680-0542-6, pp 1-22, 2000. 
[22] Cairns A, Sheppard CGW. Cyclically resolved simultaneous flame and flow imaging in a SI engine. SAE Trans., 109, J. of Engines (Section 3), 2017-35, Sept 2001, also published as SAE Paper 2000-01-2832, 2000).

[23] Abraham J, Williams FA, Bracco FV. A discussion of turbulent flame structure in premixed charges. SAE Technical Paper No. 850345, 1985.

[24] Morel T, Rackmil Cl, Keribar R, Jennings MJ. Model for heat transfer and combustion in spark ignited engines and its comparison with experiments. SAE Technical Paper No. 880198, 1988.

[25] Abdel-Gayed RG, Bradley D, Lawes M. Turbulent burning velocities: a general correlation in terms of straining rates. Proc R Soc Lond 1987;A414:389-413.

[26] Keck JC, Heywood JB, Noske G. Early flame development and burning rates in spark ignition engines and their cyclic variability. SAE Technical Paper No. 870164, 1987.

[27] Dai W, Davis GC, Hall MJ, Matthews RD. Diluents and lean mixture combustion modelling for SI engines with a quasi-dimensional model. SAE Technical Paper No. 952382, 1995.

[28] Lipatnikov AN, Chomiak J. A simple model of unsteady turbulent flame propagation. SAE Technical Paper No. 972993, 1997.

[29] Lipatnikov AN, Chomiak J. Turbulent flame speed and thickness: phenomenology, evaluation, and application in multi-dimensional simulations. Prog Energy Combust Sci 2002;28:1-74.

[30] Zimont VL. Gas premixed combustion at high turbulence. Turbulent flame closure combustion model. Exp Thermal Fluid Sci 2000;21:179-86.

[31] Hicks RA, Lawes M, Sheppard CGW, Whitaker, B.J. Multiple laser sheet imaging investigation of turbulent flame structure in a spark ignition engine. SAE Technical Paper No. 941992, 1994.

[32] Keck JC. Turbulent flame structure and speed in spark ignition engines. Proc Combust Inst 1982;19-1451-66.

[33] Blizard NC, Keck JC. Experimental and theoretical investigation of turbulent burning model for internal combustion engines. SAE Technical Paper No. 740191, 1974.

[34] Tabaczynski RJ, Ferguson CR, Radhakrishnan K. A turbulent entrainment model for spark-ignition engine combustion. SAE Technical Paper No. 770647, 1977.

[35] Hires SD, Tabaczynski RJ, Novak JM. The prediction of ignition delay and combustion intervals for a homogeneous charge, spark ignition engine. SAE Technical Paper No. 780232, 1978.

[36] Tabaczynski RJ, Trinker FH, Shannon BAS. Further refinement and validation of a turbulent flame propagation model for spark-ignition engines. Combust Flame 1980;39:111-21.

[37] Heywood JB. Combustion and its modeling in spark-ignition engines. Int. Symposium COMODIA 94, 1994.

[38] Bozza F, Gimelli A, Merola SS, Vaglieco BM. Validation of a fractal combustion model through flame imaging. SAE Technical Paper No. 2005-01-1120, 2005.

[39] Verhelst S, Woolley R, Lawes M, Sierens R. Laminar and unstable burning velocities and Markstein lengths of hydrogen-air mixtures at engine-like conditions. Proc Combust Inst 2005;30, 209-16.

[40] Bradley D, Lawes M, Liu K, Verhelst S, Woolley R. Laminar burning velocities of lean hydrogen-air mixtures at pressures up to $1.0 \mathrm{MPa}$. Combust Flame 2007;149:162-72. 
[41] Metghalchi M, Keck JC. Burning velocities of mixtures if air with methanol, iso-octane and indolene at high pressure and temperature. Combust Flame 1982;48:191-210.

[42] Ramos JI. Internal combustion engine modeling. Hemisphere Publishing Corporation, 1989.

[43] Douaud AM, Eyzat P. Four-Octane-Number Method for Predicting the Anti-Knock Behavior of Fuels and Engines. SAE Technical Paper No. 780080, 1978.

[44] Halstead MP, Kirsch LJ, Quinn CP. The autoignition of hydrocarbon fuels at high temperatures and pressures- fitting of a mathematical model. Combust Flame 1977;30:45-60.

[45] Cowart JS, Keck JC, Heywood JB, Westbrook CK, Pitz WJ. Engine knock predictions using a fully-detailed and reduced chemical kinetic mechanism. Proc Combust Inst 1990;23:1055-62.

[46] Burluka AA, Liu K, Sheppard CGW, Smallbone AJ, Woolley R. The Influence of Simulated Residual and NO Concentrations on Knock Onset for PRFs and Gasolines. SAE Technical Paper No. 2004-01-2998, 2004. 


\section{Figure captions}

Figure 1. Rapid Prototype reconstruction of a segment of a turbulent flame

Figure 2. In-cylinder velocity field with superimposed successive turbulent flame front images (details in text)

Figure 3. Mass fraction burned versus crank angle. Also shown is how the observed behaviour can be approximated with a set of equations of the form (1.9) and (1.10). 


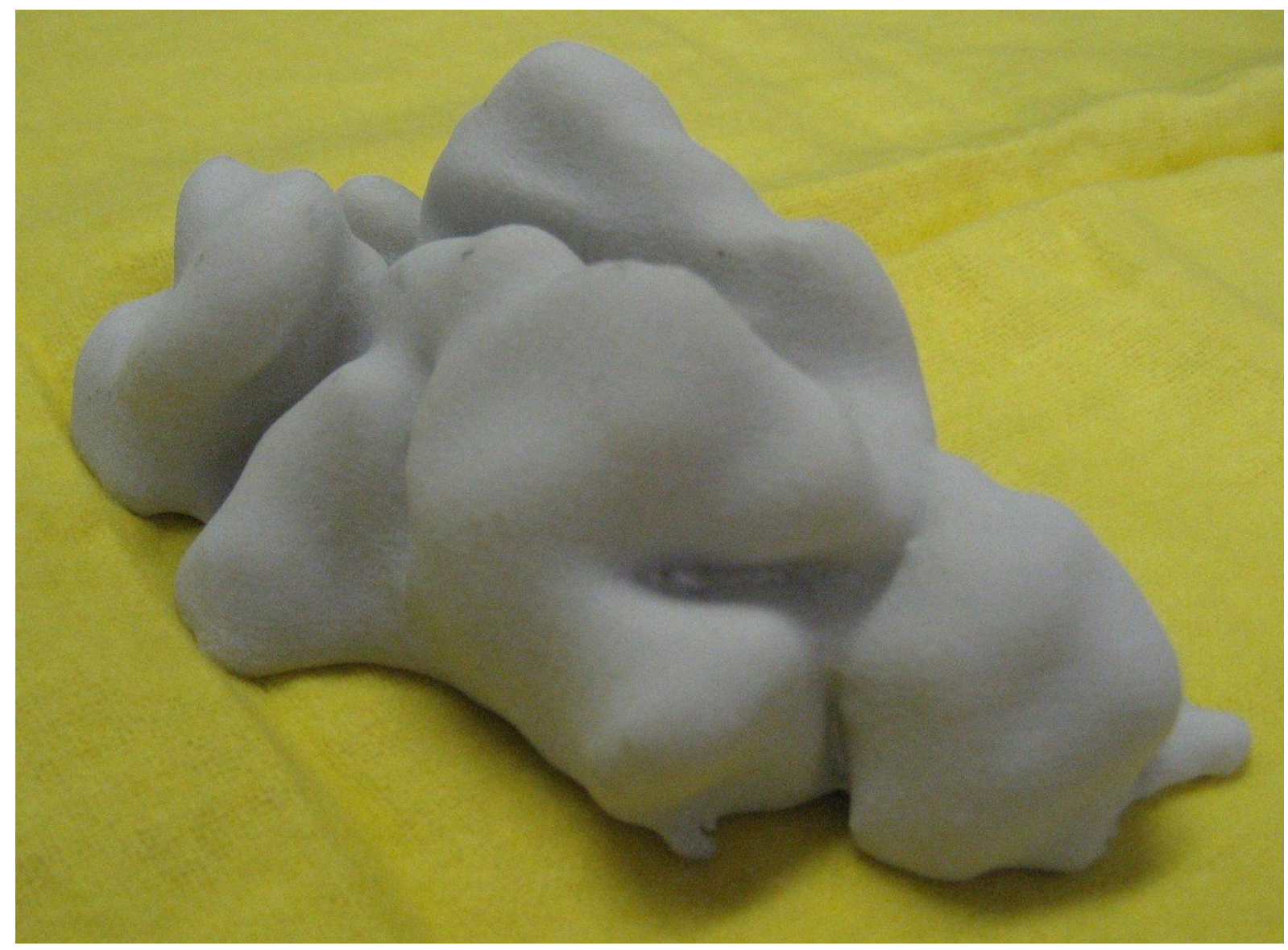

Figure 1. Rapid Prototype reconstruction of a segment of a turbulent flame 


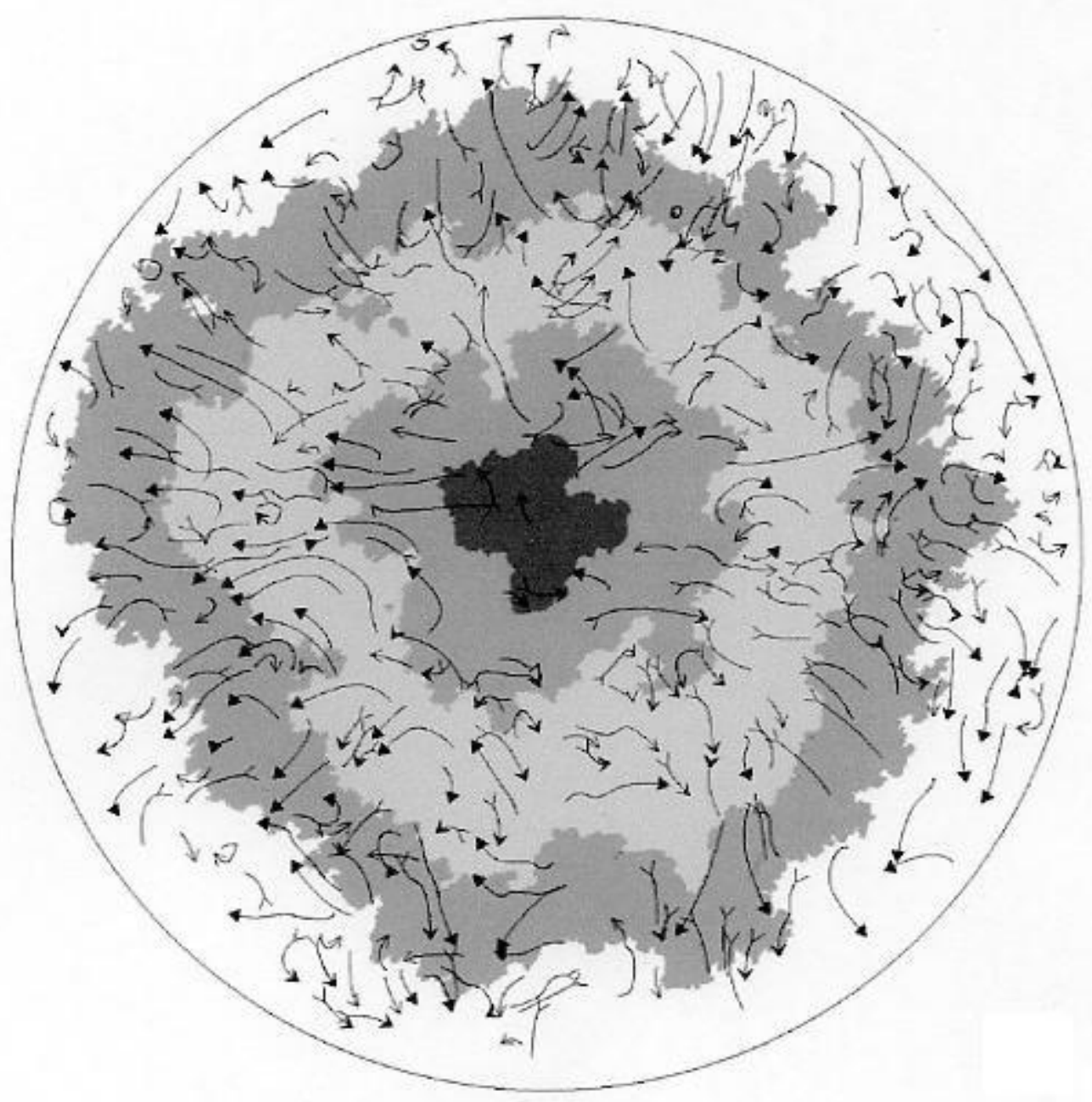

Figure 2. In-cylinder velocity field with superimposed successive turbulent flame front images (details in text) 


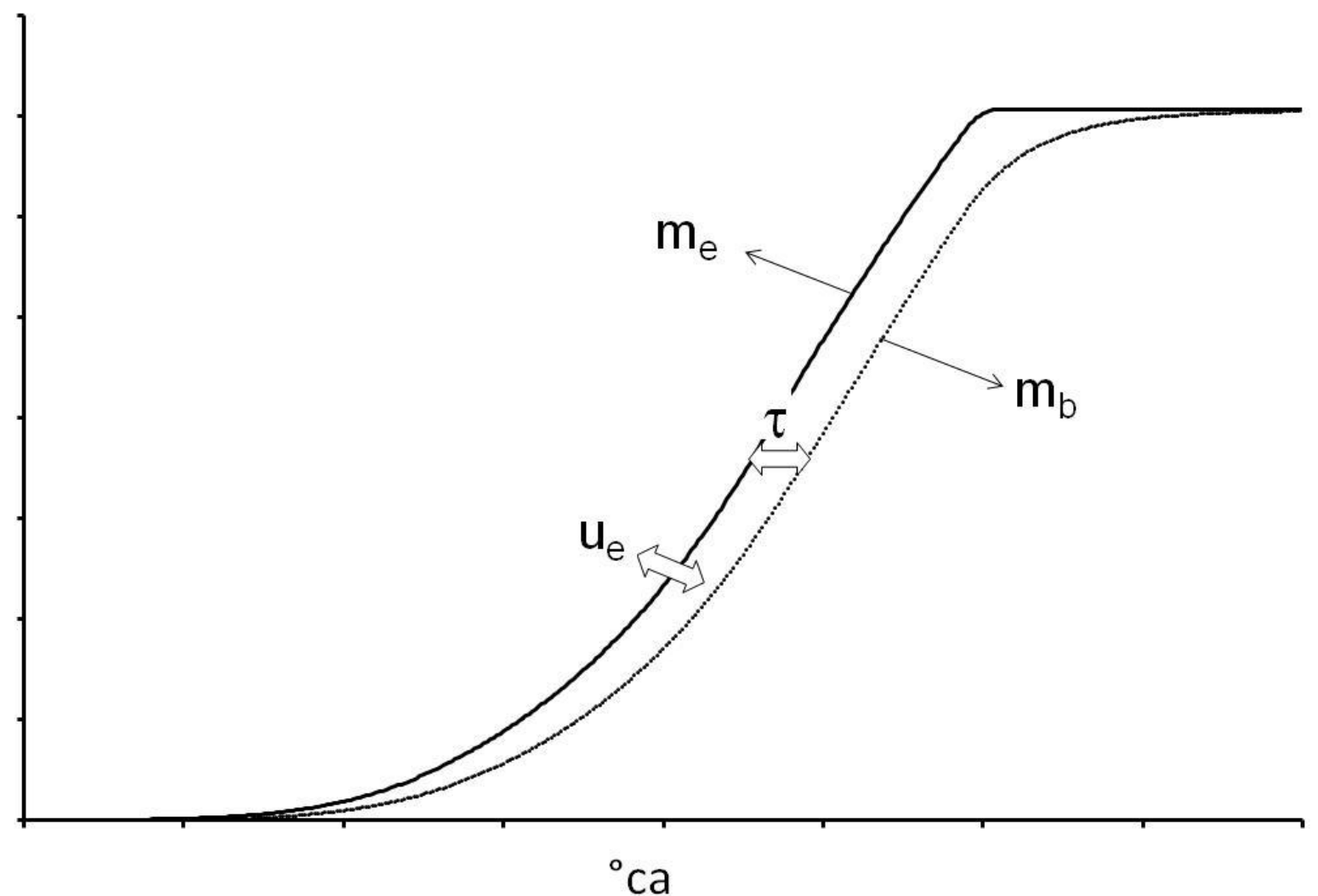

Figure 3. Mass fraction burned versus crank angle. Also shown is how the observed behaviour can be approximated with a set of equations of the form (1.9) and (1.10). 